
\title{
28 Research Suare \\ Joint Channel Assignment and Bandwidth Reservation using Improved FireFly Algorithm (IFA) in WMN
}

narayanarao narayanarao ( $\square$ narayanarao123phd@gmail.com )

NBKRIST: NBKR Institute of Science and Technology

\section{A. Rajasekhar Reddy}

NBKRIST: NBKR Institute of Science and Technology

\section{Research Article}

Keywords: WMN, network, Congestion, IFA, channel

Posted Date: June 28th, 2021

DOl: https://doi.org/10.21203/rs.3.rs-486592/v1

License: (c) (1) This work is licensed under a Creative Commons Attribution 4.0 International License.

Read Full License 


\title{
Joint Channel Assignment and Bandwidth Reservation using Improved FireFly Algorithm (IFA) in WMN
}

\author{
Dr. A. Narayana Rao \\ Assoc Professor,CSE Department, NBKRIST, \\ Vidyanagar,India \\ narayanarao123phd@gmail.com \\ Dr. A. Rajasekhar Reddy, \\ Professor,CSE Department,NBKRIST, \\ Vidyanagar,India. \\ arsreddy521@gmail.com
}

\begin{abstract}
In WMN, at the time of network consignment and bandwidth registration, the active network consignment method did not take into consideration the intrusion, congestion load and bandwidth necessities as a whole. The significance centred bandwidth registration methods result in famishment of slightest significance congestion. Hence in this paper, we propose a Joint Channel Assignment and Bandwidth Reservation using Improved FireFly Algorithm (IFA) in WMN. Initially the priority of each node is determined based on the channel usage, future interference and link congestion probability metrics. The bandwidth is allocated straight, comparative to the nodule significance and entire quantity of congestion movements incomplete on the demanded nodule. For channel assignment and path selection, the improved FireFly Algorithm (IFA) is used. The objective function of IFA is determined in terms of link capacity, interference and flow conservation constraints. Then the channels and the path which minimize the objective function are selected by applying IFA. By simulation results we show that the proposed technique minimizes the traffic and enhances the channel efficiency.
\end{abstract}

Keywords: WMN; network; Congestion; IFA; channel

\section{Introduction}

Wireless Mesh Network (WMN) is a network with nodules which are associated to one another with no wires devoid of any set-up. The wireless mesh network is taken into consideration as the most effectual communication method to deal IP connectivity, as it bids haste IP link to an enormous series. WMN is made of mesh clients (MCs) and wireless mesh routers (WMRs) which depend upon one another's packages in a multihop way. WMRs have negligible movement and perform as the support of WMNs. WMN has certain distinct WMRs, known as Internet Gateways (IGWs) which is able to perform as communication bonds amid the Internet and BWMN. Besides, IGWs offer Internet availability [1]. WMNs are stimulated in the manufacturing customary clusters, like IEEE 802.11, IEEE 802.15, and IEEE 802.16. Certain solicitations of WMN are to admit broadband internet, inside WLAN, mobile user admission and connectivity [2].

The notion of channel consignment is to curtail the network-wide intrusion by exploiting non-overlapping networks in wireless broadcasts. The objective of network consignment is to enhance the presentation of the network specifically when network volume is considered. The chief task is passing on the existing networks in such a way that intrusion is diminished when confirming the network connectivity. Likewise, the networks ought to be allotted to the network boundaries with regard to the specified predictable load on every simulated connection.

Channel assignment procedures are able to be unified or dispersed. Unified channel assignment methods rely on a chief unit known as channel assignment server (CAS) which does channel assignment for the entire network centered on the universal network sight. In dispersed channel assignment methods, the procedure goes on each network nodule and implements channel assignment choices bearing in mind only limited info into 
interpretation. Channel assignment methods are categorised as active or inert. In active methods, channel shifts may happen regularly while inert methods in disparity, shift the borders to a specific channel for an extended time [3][4].

A vital issue in networking is the distribution of restricted sources amongst the consumers of a network. Bandwidth takes a vital part in WMN load harmonising. The constraint of WMN is chiefly because of the restricted bandwidth collected with network volume. Bandwidth arrangement aids in giving QoS assured amenities.

\subsection{Motivation and Objectives}

Both static and dynamic channel allocation strategies have disadvantages [1]. Channel distribution procedures intensely rely on the intrusion prototype and the network congestion weight.

Load-aware channel assignment systems may possibly not be appropriate when weight varies fast and probably ought to apprise consequently. The POCA algorithm [8] considers only interference metric for channel assignment ignoring the traffic load and bandwidth requirements of flows. In Adaptive Dynamic Channel Allocation (ADCA) protocol [7], least congested channels are assigned. But it does not present any strong approach for bandwidth reservation based on these channels.

If the sum of bandwidth set aside is smaller than the real congestion weight, then the probabilities of losing data or misrepresentation of the data is additional. Conversely, if the congestion weight is pointedly minor, then the supplementary bandwidth will be lost. In [2], the bandwidth is allocated based on traffic load and interference using fair end-to-end bandwidth allocation (FEBA). But it introduces starvation of low priority traffic.

Hence we can summarize the problems of existing works on channel assignment and bandwidth reservation as follows:

- The dynamic channel assignment technique did not consider the interference, traffic load and bandwidth requirements together.

- The priority based bandwidth reservation techniques lead to starvation of least priority traffic.

- The bandwidth reservation technique was not aware of the interference free channels

Hence the main objective of the research work is design a joint channel assignment and bandwidth reservation technique which should

- Minimize the interference in the network

- Minimize the channel switching delay

- Avoid congestion in network

- Ensure effective bandwidth utilization for all traffic flows

Based on the identified drawbacks, a joint channel assignment and bandwidth reservation technique will be proposed.

\section{Related Works}

Sanjeev Jain et al [2] have familiarised a traffic load and interference based bandwidth allocation (TLIBA) system for wireless mesh network (WMN) which enhances the suspension and output presentation by correct use of allotted bandwidth. The bandwidth is allotted depending on traffic load and interference together. Then an appropriate way is chosen depending upon the smallest routing metric (RM) charge.

Amjad Ali et al [5] have offered a new Integer Linear Programming (ILP) optimization prototype to renovate the ideal linked mesh support topology with a least amount of associates and transmit nodules that gratifies the provided endwise QoS requests for hypermedia congestion and documentation of additional sources, while 
upholding idleness. They also suggested a polynomial period experiential procedure known as Link and Node Removal Considering Residual Capacity and Traffic Demands (LNR-RCTD).

DEGAN ZHANG et al [6] have offered MRMC source optimization technique centred on curved concept for WMN. As stated by curved concept, they utilized the curved optimization purpose to acquire the ideal result in the restricted network assisted by Ad hoc On-Demand Distance Vector (AODV) routing way. Consequently, they determined the ideal result into three modest sub-problems based upon the Lagrange dichotomy technique assisted by multi-radio multi-channel AODV (MAODV)

Yong Ding et al [7] have projected a fusion multichannel multiradio wireless mesh networking design, in which every mesh nodule has both inert and active borders. Initially they offered an Adaptive Dynamic Channel Allocation procedure (ADCA), which takes into consideration, the optimization for both output and suspension in the channel consignment. Besides, they also suggested an Interference and Congestion Aware Routing procedure (ICAR) in the fusion network with both inert and active links, which poises the channel utilisation in the network.

Jihong Wang et al [8] have suggested a congestion-irrelevant network consignment procedure that allots networks for the entire associations in the system when reducing the entire system intrusion. Speculative estimation method is exploited to gain the straight association amid intrusion varieties and network split-ups that is able to be effortlessly smeared to mesh networks with numerous formations deprived of alteration. Since the congestion amid the Internet and customers is measured to be leading, space from the entry, quantity of adjoins and intrusion are cast-off to define the network consignment sequence of associations.

Mansoor Alicherry et al [10] have proposed an Efficient channel assignment and routing for throughput optimization of mesh clients. Efficient channel assignment schemes can greatly relieve the interference effect of close-by transmissions; effective routing schemes can alleviate potential congestion on any gateways to the Internet, thereby improving per-client throughput. Unlike previous heuristic approaches, we mathematically formulate the joint channel assignment and routing problem, taking into account the interference constraints, the number of channels in the network and the number of radios available at each mesh router. They then used this formulation to develop a solution for our problem that optimizes the overall network throughput subject to fairness constraints on allocation of scarce wireless capacity among mobile clients.

Jasmine David et al [11] have proposed Priority Based Interference Aware Bandwidth Reservation (PBIABR) and Interference Aware Bandwidth Reservation (IABR) for many flowing rates under dynamic scenario. Channel Priority plays a vital role to opt the channel which posses less interference for efficient bandwidth reservation for PBIABR. The opted channel will have the minimal channel interference effect. IABR posses the controllability character for data flow to establish end-to-end communication over Multi-Radio Multi-Channel (MRMC) - Wireless Mesh Network. Their research paper focuses on deep analysis of Residual Energy (joule) compared with Interval (sec) and Average Energy (joule) compared with Interval (sec) under dynamic scenario for multiple flow rates by implementing PBIABR and IABR criteria.

Hamed Mohsenian Rad et al [12] have formulated the Joint Optimal Channel Assignment and Congestion Control (JOCAC) as a decentralized utility maximization problem with constraints that arise from the interference of the neighboring transmissions. Unlike other previous work, the JOCAC algorithm is able to assign not only the non-overlapping (orthogonal) channels, but also the partiallyoverlapping channels within the IEEE 802.11 frequency bands. Using $802.11 \mathrm{~b}$ with 3 non-overlapping channels, simulation results show that our algorithm provides a higher aggregated goodput than the recently proposed load-aware algorithm by $20 \%$. The goodput is further increased by $40 \%$ when all the partially-overlapping channels are being used.

\section{Joint Channel Assignment and Bandwidth Reservation using IFA (JCABR-IFA)}

\subsection{Overview}


Initially the priority of each node is determined based on the channel usage, future interference and link congestion probability metrics. The bandwidth then allotted is openly proportionate to the importance of the nodule and overall quantity of congestion movements incomplete on the demanded nodule.

For channel assignment and path selection, the improved FireFly Algorithm (IFA) is used. The objective function of IFA is determined in terms of link capacity, interference and flow conservation constraints. Then the channels and the path which minimize the objective function are selected by applying IFA.

\subsection{Node Priority Assignment}

Based on the obtained parameter values, each node computes a priority level as

$$
\mathrm{W}=\mathrm{a}_{1} * \mathrm{IR}+\mathrm{a}_{2} * \operatorname{Pr}+\mathrm{a}_{3} * \mathrm{CU}
$$

Where $\mathrm{a}_{1}, \mathrm{a}_{2}, \mathrm{a}_{3}$ are randomly chosen weight factors

Here $\mathrm{CU}$ is the channel usage.

IR is the interference range on the adjacent channels which is defined using the following equation:

$\mathrm{IR}=\sqrt[n]{U_{t} \cdot V_{t} \cdot V_{r} \cdot w_{t}^{2} \cdot w_{r}^{2} \cdot o p(i, j)} / F_{t h}$

$\mathrm{U}_{\mathrm{t}}$ is transmission power at sender node

$V_{t}$ and $V_{r}$ be the antenna gains of the sender and receiver

$\mathrm{W}_{\mathrm{t}}$ and $\mathrm{w}_{\mathrm{r}}$ be the height of the antennas

$\mathrm{n}$ is path loss parameter

op is overlap between channel $\mathrm{i}$ and $\mathrm{j}$

$F_{\text {th }}$ is carrier sensing threshold

Pr is the Link congestion probability which is defined as follows:

$$
\operatorname{Pr}_{\mathrm{c}}=\frac{z(k)}{q(k)}
$$

$z(k)$ is the numeral of adjoins for any of the two terminal ends

$\mathrm{q}(\mathrm{k})$ is the minimum hop count distance from the gateway, for each link $\mathrm{k}$.

If $\mathrm{W}$ is high, then node is assigned a higher priority. The mass rates are supposed to be great when IR, $\mathrm{Pr}$ and $\mathrm{CU}$ are fewer than the verge rate. Though there exist some gap in any one of the factor verge, then the nodule stays to announce itself with greater precedence.

\subsection{Bandwidth Assignment}

The allocated bandwidth is openly proportionate to the nodule significance and overall sum of congestion movements incomplete on the demanded nodule. 


$$
\mathrm{BW}_{\mathrm{i}}=\frac{\sum_{j \in R} P_{j} * G_{i}(j)}{\sum_{j \in R} P_{j}}+P T
$$

where $P_{\mathrm{j}}$ refers to the node priority

$G_{i}(j)$ refers to indicator which equals to 1 if $j$ is under service at queue $i, 0$ otherwise.

PT refers to traffic interference

$$
\mathrm{PT}=\mathrm{ETT}_{\mathrm{i}} * \mathrm{AL}_{\mathrm{i}}
$$

where ETT is the difference in transmission rate and loss ratio of links

$\mathrm{AL}_{\mathrm{i}}$ is the average load

\subsection{IFA based Channel Assignment and Path Selection}

Firefly Algorithm (FA) is regarded as the one among the meta-heuristic method built to resolve optimization problems utilizing the simulation of behavior of the fireflies. The algorithm derives its inspiration from the flashing lights of fire-flies in nature. It consists of three kinds of fireflies: searching firefly, listening firefly, and updating firefly.

Attractiveness depends on the brightness and distance between the firefly. So one firefly moves forward to the next, based on the attraction between them. [9].

Let $\mathrm{G}, \mathrm{H}$ and $\mathrm{M}$ be the searching, listening and updating firefly respectively.

\subsubsection{Objective function of IFA}

For channel assignment and path selection, the improved FireFly Algorithm (IFA) is used.

The objective function of IFA is determined in terms of link capacity, interference and flow conservation constraints.

Link capacity constraint: The totality of the entire movements on any connection $\mathrm{m}_{\mathrm{ij}}$ necessarily is fewer than or equivalent to the entire allocated size to that connection: It is given by Eq. (6)

Flow Conservation Constraint: The amount of the entire movements on some connection $\mathrm{m}_{\mathrm{i}, \mathrm{j}}$ which is to be detached need to be fewer than or equivalent to the amount of remaining dimensions over the entire occurrence associations. It is given by Eq. (7).

Interference: Interference series prototype tells that connection $\mathrm{m}_{\mathrm{i}, \mathrm{j}}$ amid nodules $\mathrm{N}_{\mathrm{i}}$ and $\mathrm{N}_{\mathrm{j}}$, and connection $\mathrm{m}_{\mathrm{k}, \mathrm{l}}$ amid nodules $\mathrm{N}_{\mathrm{k}}$ and $\mathrm{N}_{\mathrm{l}}$ possibly will not be allocated the similar network if the dispatcher or recipient of any of them is in the intrusion series of the dispatcher or recipient of the additional one.

After that the networks and the track that diminish the target purpose are designated by putting on IFA.

Minimize $\mathrm{O}=\sum_{T_{0} \in T} \sum_{m_{i, j} \in M} \sum_{C_{t} \in C} E\left(m_{i, j}, C_{t}\right)$

Subject to the following constraints: 
$\sum_{T_{0} \in T} E\left(m_{i, j}, \mathrm{C}_{\mathrm{t}}\right) * \mathrm{~T}_{0} * \mathrm{~s}_{(\mathrm{i}, \mathrm{j})} \mathrm{t} \leq \mathrm{AC}_{\mathrm{mi}, \mathrm{j}}$

$\sum_{T_{0} \in T} E\left(m_{i, j}, \mathrm{C}_{\mathrm{t}}\right) * \mathrm{~T}_{0} * \mathrm{~s}_{(\mathrm{i}, \mathrm{j})} \mathrm{t}-\sum_{m_{i, j} \in G_{i}} E\left(m_{i, j}, \mathrm{C}_{\mathrm{t}}\right) * \mathrm{RES}_{m(i, j)} \leq 0$

$\forall m_{i, j} \in M$ and $C_{t} \in C$

where,

$\mathrm{T}_{0}=$ The sum of dimensions utilized by congestion movement

$\mathrm{C}_{\mathrm{t}}=$ total number of channels

$\mathrm{s}_{\mathrm{ij}}$ - static channel allocation

$\mathrm{AC}=$ total assigned capacity to link $\mathrm{m}_{\mathrm{i}, \mathrm{j}}$

\subsubsection{IFA Algorithm}

Consider the following parameters

$\mathrm{B}$ be the population size

$\mathrm{O}_{\mathrm{b}}$ be the target proportion of result I related to finest result

$\mathrm{O}_{\mathrm{w}}$ be the target proportion of the entire populace related to finest result

$\beta_{r n 1}, \beta_{r n 2}$ Arbitrary results amongst the existing populace

$\beta_{j}$ is the solution with better fitness than $\beta_{i}$

The steps involved in this algorithm are as follows:

1. Initialize the population B in random manner

2. Calculate the objective function of the whole population $\mathrm{O}_{\mathrm{i}}(\mathrm{i}=1,2, \ldots, \mathrm{B})$

3. Determine the best solution $\beta_{\text {best }}$ and the worst solution $\beta_{\text {worst }}$

4. Set current iteration $\gamma=0$

While $\gamma<\mathrm{N} \gamma$

Set $\gamma=\gamma+1$

Calculate $\mathrm{O}, \mathrm{O}_{\mathrm{b}}$ and $\mathrm{O}_{\mathrm{w}}$

For $\mathrm{i}=1$ : $\mathrm{B}$

For $\mathrm{j}=1$; $\mathrm{B}$ 
If $\mathrm{O}_{\mathrm{i}}>\mathrm{O}_{\mathrm{j}}$ Then

Determine $\Delta \beta_{1 i j}$ and $\Delta \beta_{2 i j}$

$$
\Delta \beta_{1 i j}=\left(\beta_{\text {best }}-\beta_{\text {worst }}\right)
$$

$$
\Delta \beta_{2 i j}=\left(\beta_{j}-\beta_{i}+\beta_{m 1}-\beta_{r n 2}\right)
$$

If $\mathrm{O}_{\mathrm{b}}>\mathrm{O}_{\mathrm{w}}$ Then

$$
\Delta \beta_{i j}=\Delta \beta_{1 i j}
$$

Else

$$
\Delta \beta_{i j}=\Delta \beta_{2 i j}
$$

End

5. Estimate radius $\mu_{i b e t}$

$$
\mu_{i b e t}=\sqrt{\left(\delta_{i}-\delta_{b e s t}\right)^{2}}
$$

6. Estimate attractiveness $\sigma$ and new solution as follows:

$$
\begin{aligned}
& \sigma=\sigma_{0} e^{-\lambda r_{i, j}^{2}} \\
& \beta_{i j}^{\text {new }}=\beta_{i}+\alpha \cdot r n \cdot \Delta \beta_{i j}-r n
\end{aligned}
$$

7. Calculate the objective function of $\beta_{i j}^{\text {new }}$

8. Compare each old and new solution to keep the better one

9. Compare the objective function to choose the best objective function

10. The channels and the path which minimize the objective function are selected by applying IFA.

\section{Simulation Results}

\subsection{Simulation Parameters}

The simulation of JCABRIFA is conducted in Ns-2 and it is compared with TLIBA. The performance is evaluated with respect to Bandwidth, Fairness, E2D, PDR, Packet Drop and throughput.

Various simulation settings are listed in table 1. 


\begin{tabular}{|l|l|}
\hline No. of Nodes & 53 \\
\hline Area & 1300 X 1300 \\
\hline MAC & 802.11 \\
\hline Simulation Time & $50 \mathrm{sec}$ \\
\hline Traffic Source & CBR \\
\hline Rate & $100,200,300,400$ and $500 \mathrm{~Kb}$ \\
\hline Propagation & TwoRayGround \\
\hline Antenna & OmniAntenna \\
\hline Packet Size & 512 bytes \\
\hline
\end{tabular}

\section{A. Based on Rate}

In our first experiment we vary the transmission rate as 100,200,300,400 and 500Kb.

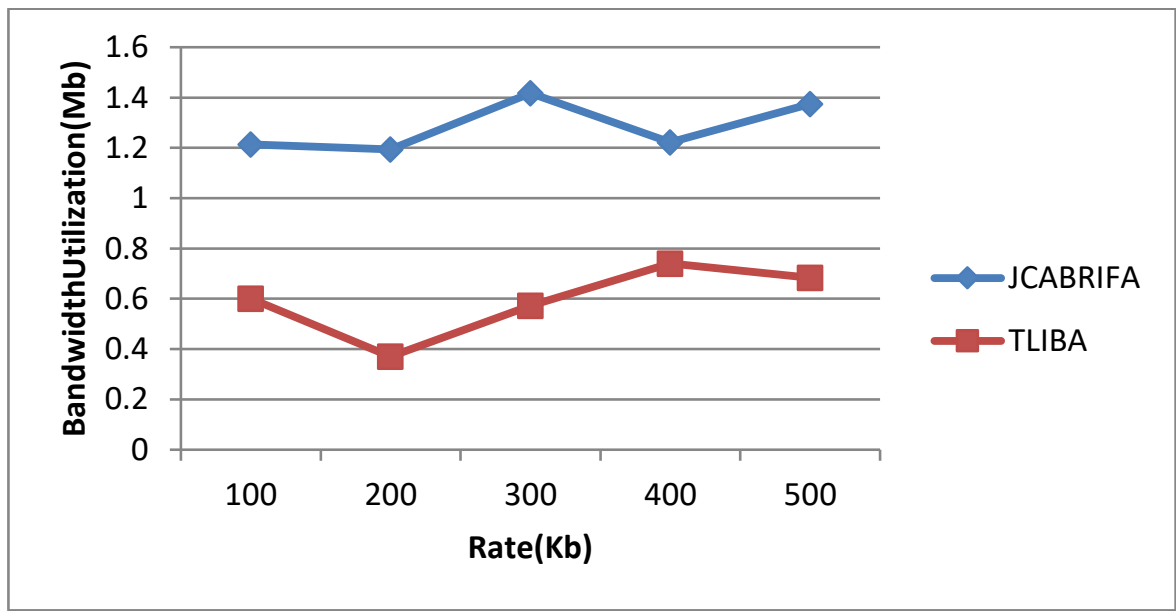

Figure 1: Bandwidth for varying Rate

The graph showing the results of Bandwidth utilization for varying the rate, is shown in Figure 1. The figure depicts that the Bandwidth utilization of JCABRIFA ranges from 1.2 to $1.3 \mathrm{Mb} / \mathrm{s}$ and Bandwidth utilization of CCARS ranges from 0.6 to 0.68 . Ultimately, the Bandwidth utilization of JCABRIFA is $54 \%$ higher when compared to TLIBA. 


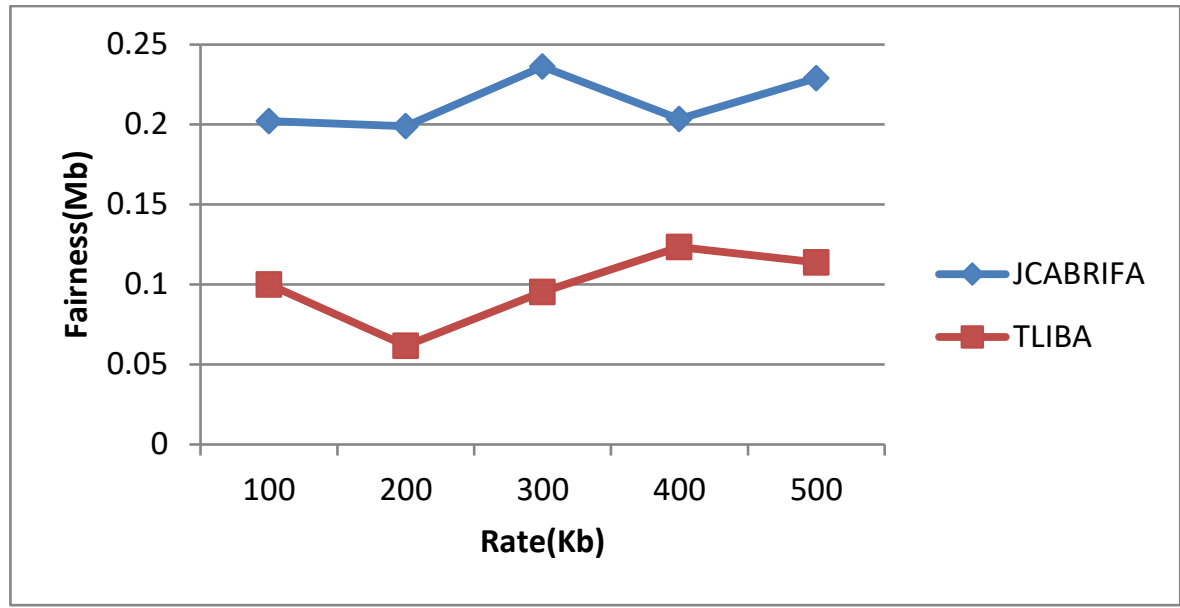

Figure 2: Fairness for varying Rate

The graph showing the results of fairness for varying the rate, is shown in Figure 2. The figure depicts that the fairness of JCABRIFA ranges from 0.20 to $0.22 \mathrm{Mb} / \mathrm{s}$ and fairness of CCARS ranges from 0.10 to 0.11 . Ultimately, the fairness of JCABRIFA is $54 \%$ higher when compared to TLIBA.

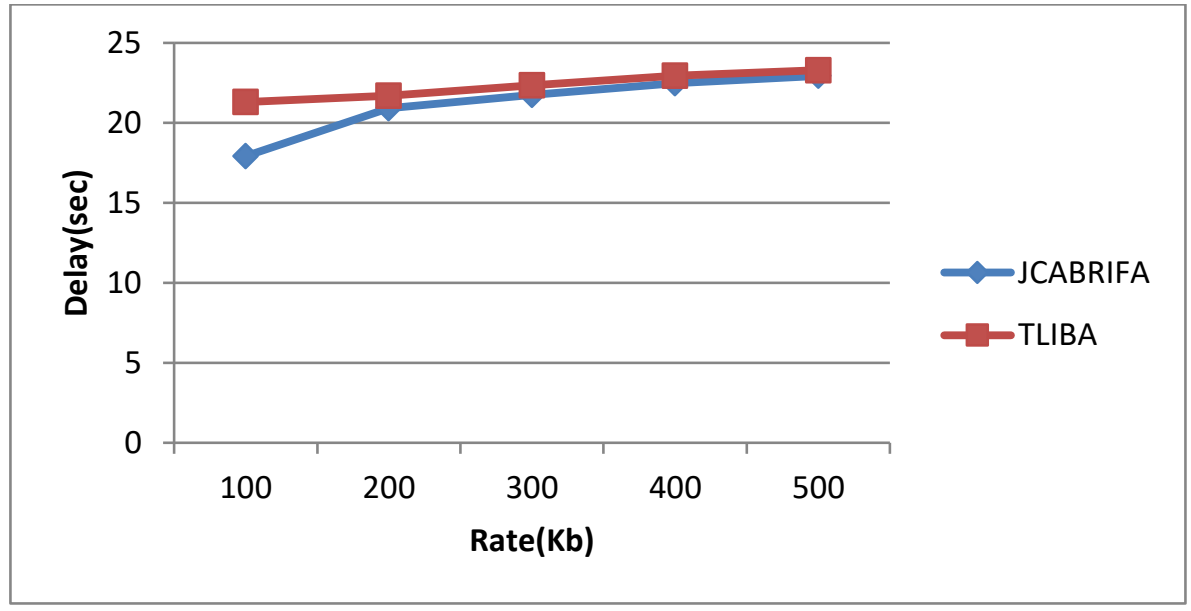

Figure 3: E2D for varying Rate

The graph showing the results of E2D for varying the rate, is shown in Figure 3. The figure depicts that the E2D of JCABRIFA ranges from 17.9 to 22.9 seconds and E2D of CCARS ranges from 21.3 to 23.2 seconds. Ultimately, the E2D of JCABRIFA is 5\% less when compared to TLIBA. 


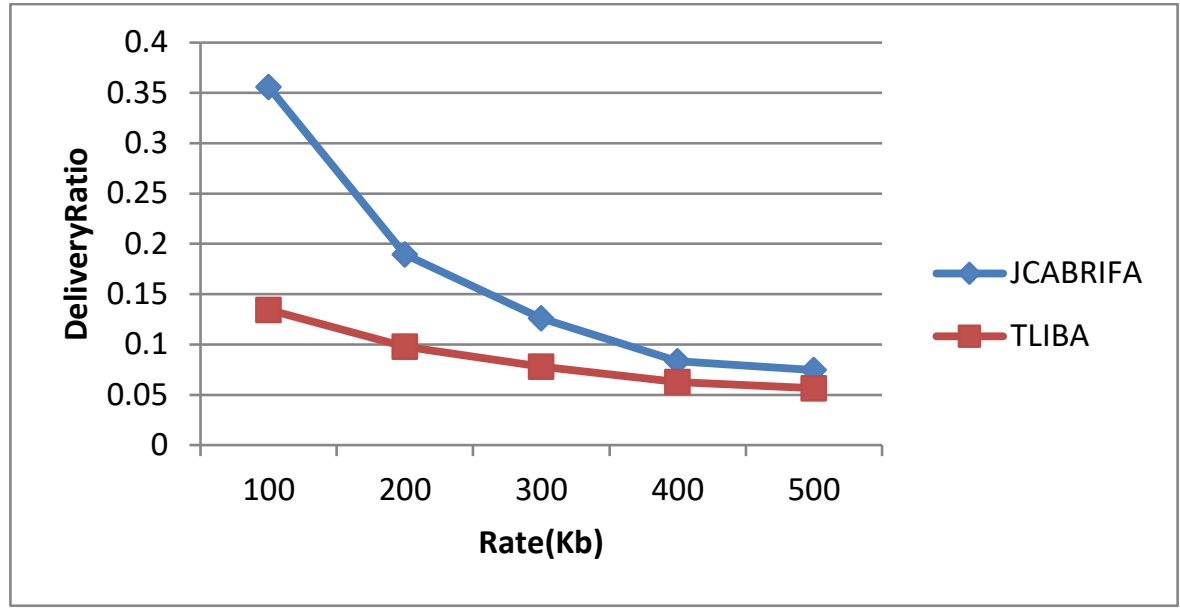

Figure 4: PDR for varying Rate

The graph showing the results of E2D for varying the rate, is shown in Figure 4. The figure depicts that the E2D of JCABRIFA ranges from 0.35 to 0.07 and E2D of CCARS ranges from 0.13 to 0.05 . Ultimately, the E2D of JCABRIFA is $39 \%$ higher when compared to TLIBA.

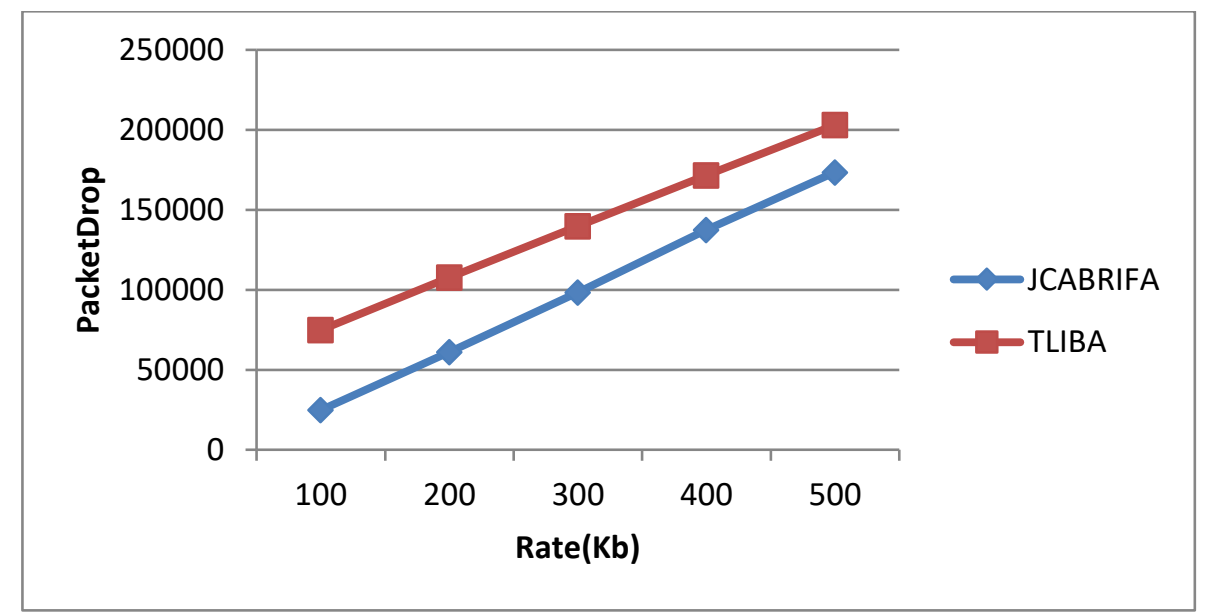

Figure 5: Packet Drop for varying Rate

The graph showing the results of Packet drop for varying the rate, is shown in Figure 5. The figure depicts that the Packet drop of JCABRIFA ranges from 24763 to 173513 seconds and packet drop of CCARS ranges from 74838 to 202979 seconds. Ultimately, the packet drop of JCABRIFA is 35\% less when compared to TLIBA.

\section{Scen-2}

\section{A. Based on Rate}

In our first experiment we vary the transmission rate as $50,100,150,200$ and $250 \mathrm{~Kb}$. 


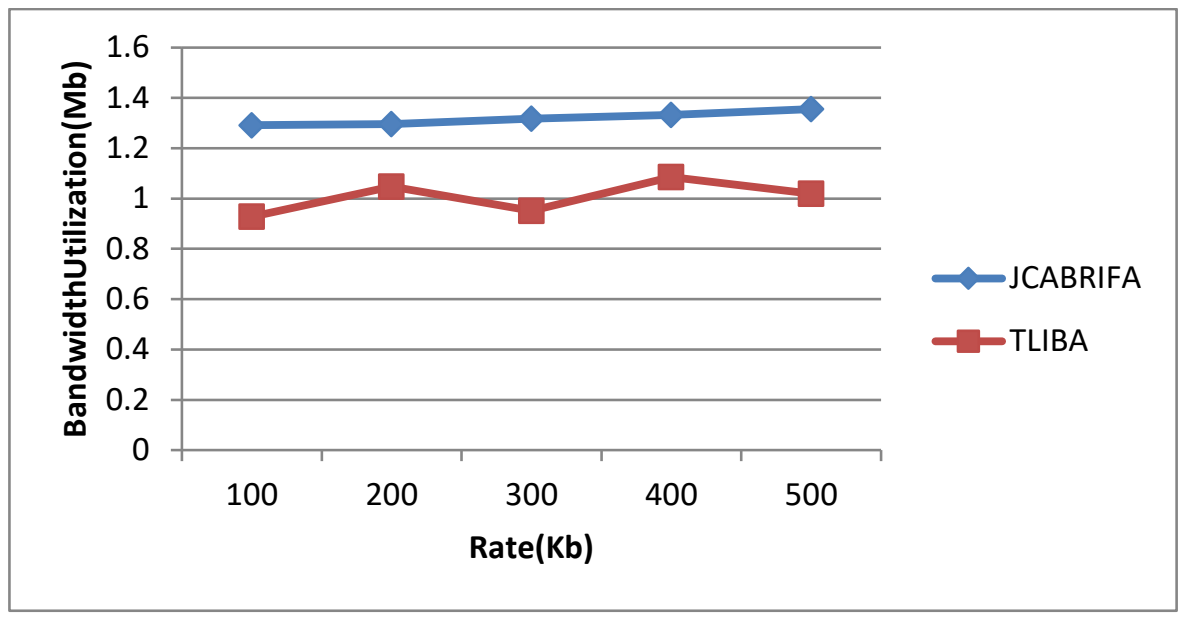

Figure 6: Bandwidth for varying Rate

The graph showing the results of bandwidth utilization for varying the rate, is shown in Figure 6. The figure depicts that the bandwidth utilization of JCABRIFA ranges from 1.2 to 1.3 seconds and bandwidth utilization of CCARS ranges from 0.92 to 1.0 seconds. Ultimately, the bandwidth utilization of JCABRIFA is $24 \%$ higher when compared to TLIBA.

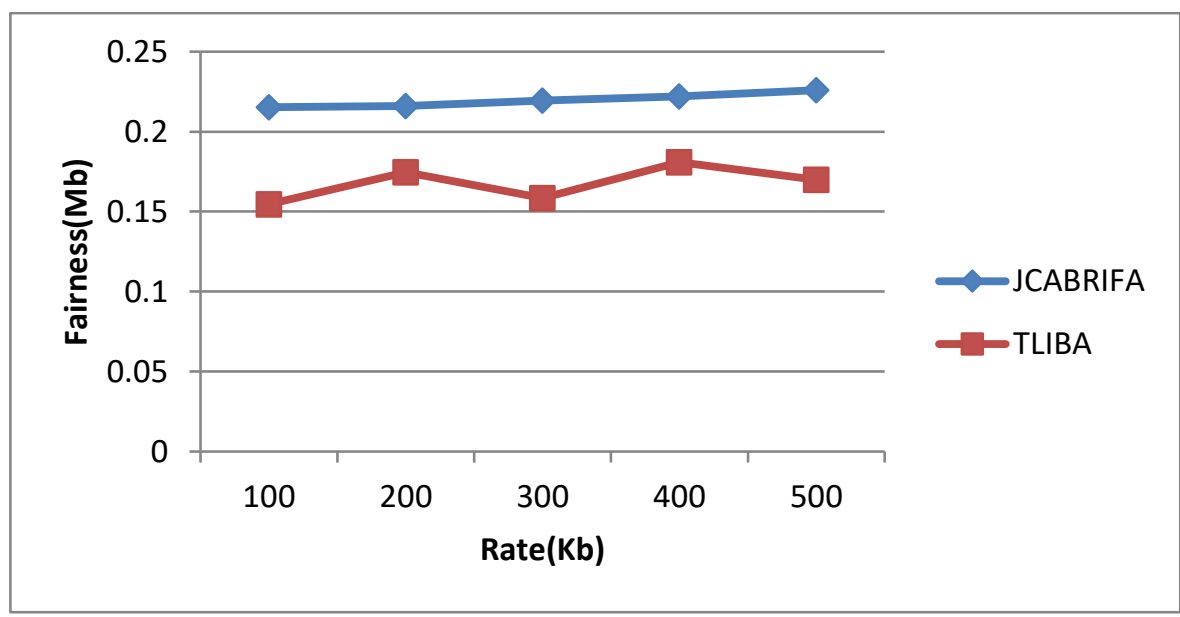

Figure 7: Fairness for varying Rate

The graph showing the results of fairness for varying the rate, is shown in Figure 7. The figure depicts that the fairness of JCABRIFA ranges from 0.21 to $0.22 \mathrm{Mb} / \mathrm{s}$ and fairness of CCARS ranges from 0.15 to 0.16 . Ultimately, the fairness of JCABRIFA is $24 \%$ higher when compared to TLIBA. 


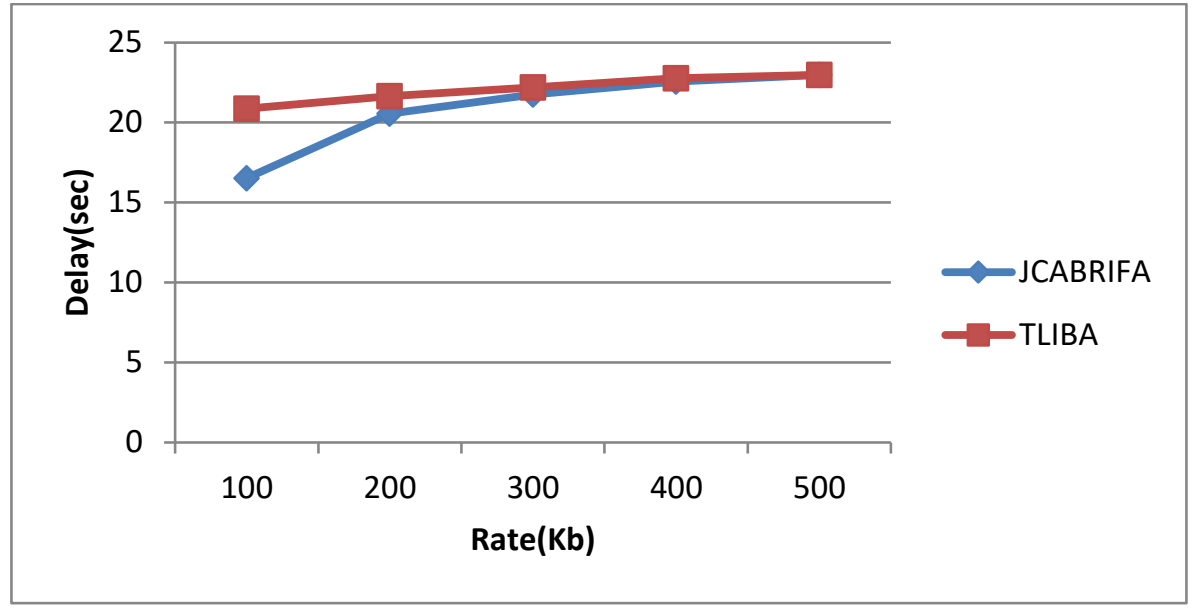

Figure 8: E2D for varying Rate

The graph showing the results of E2D for varying the rate, is shown in Figure 8. The figure depicts that the E2D of JCABRIFA ranges from 16.5 to 22.9 seconds and E2D of CCARS ranges from 20.8 to 22.9 seconds. Ultimately, the E2D of JCABRIFA is $6 \%$ less when compared to TLIBA.

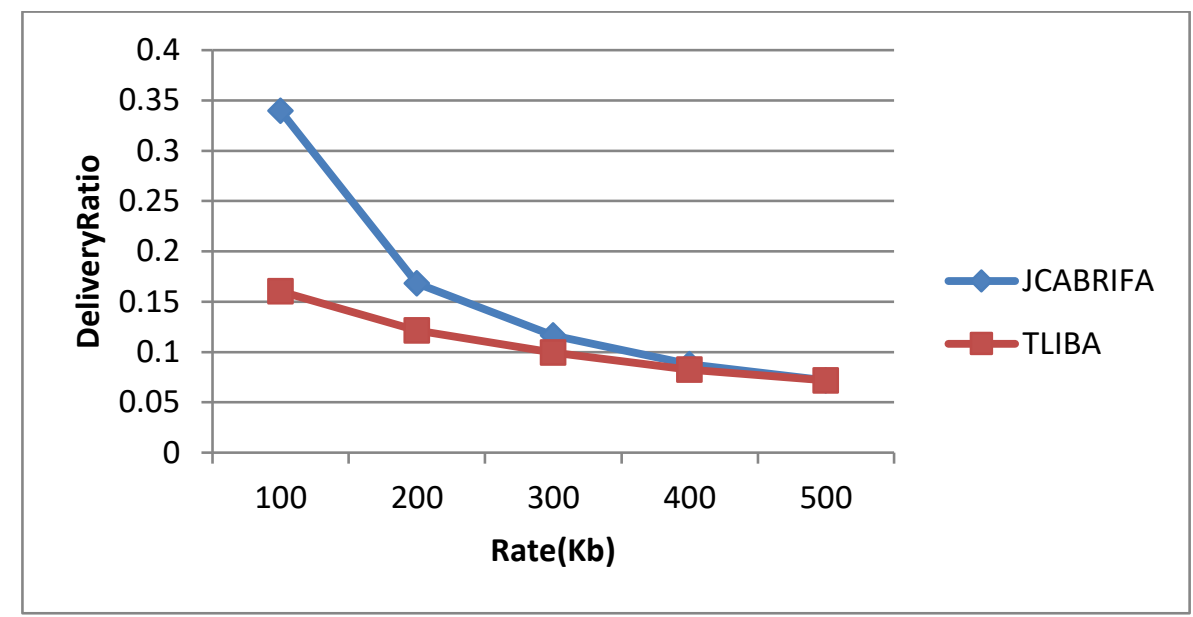

Figure 9: PDR for varying Rate

The graph showing the results of E2D for varying the rate, is shown in Figure 9. The figure depicts that the E2D of JCABRIFA ranges from 0.33 to 0.07 and E2D of CCARS ranges from 0.16 to 0.07 . Ultimately, the E2D of JCABRIFA is $20 \%$ higher when compared to TLIBA. 


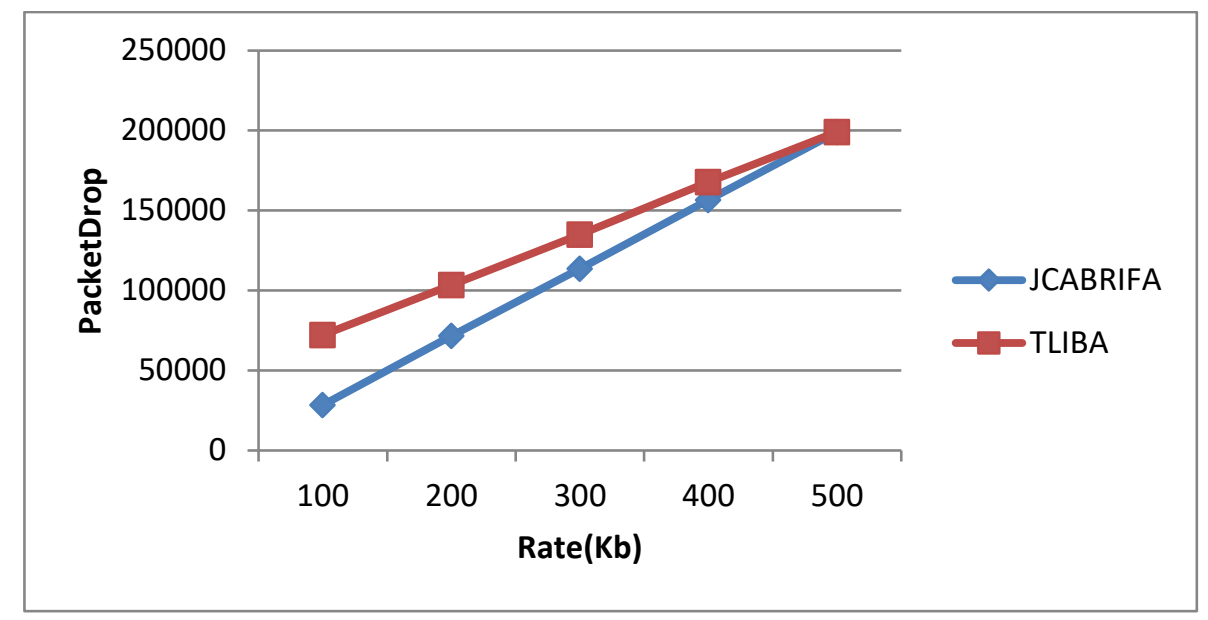

Figure 10: Packet Drop for varying Rate

The graph showing the results of Packet drop for varying the rate, is shown in Figure 10. The figure depicts that the Packet drop of JCABRIFA ranges from 28420 to 199144 seconds and packet drop of CCARS ranges from 72198 to 199144 seconds. Ultimately, the packet drop of JCABRIFA is $23 \%$ less when compared to TLIBA.

\section{Conclusion}

In this paper, we have proposed a Joint Channel Assignment and Bandwidth Reservation using Improved FireFly Algorithm (IFA) in WMN. Initially the priority of each node is determined based on the channel usage, future interference and link congestion probability metrics. The band width is assigned in then directly proportional to the node priority and total number of traffic flows pending on the requested node. For channel assignment and path selection, the improved FireFly Algorithm (IFA) is used. The objective function of IFA is determined in terms of link capacity, interference and flow conservation constraints. Then the channels and the path which minimize the objective function are selected by applying IFA. By simulation results, we have shown that the proposed technique minimizes the traffic and enhances the channel efficiency.

\section{$\underline{\text { Declaration }}$}

Funding: This research was not supported by any funding agency.

Conflict of Interest: The authors don't have any conflict of Interest

Data Availability: The authors don't use any third party data materials

Code Availability: The implementation code for this research was available with the authors.

\section{References}

1. Yong Ding and Li Xiao," Channel allocation in multi-channel wireless mesh networks", Computer Communications, Elsevier, 2011, Volume 34, pp.803-815 
2.Sanjeev Jain, Vijay Shanker Tripathi and Sudarshan Tiwari, "Bandwidth Allocation Based on Traffic Load and Interference in IEEE 802.16 Mesh Networks", Hindawi Publishing Corporation ,Journal of Engineering,2013.

3. Habiba Skalli, Samik Ghosh, Sajal K. Das, Luciano Lenzini and Marco Conti," Channel Assignment Strategies for Multiradio Wireless Mesh Networks:Issues and Solutions" IEEE Communications Magazine, 2007

4. Bong-Jun Ko, Vishal Misra, Jitendra Padhye and Dan Rubenstein," Distributed Channel Assignment in Multi-Radio 802.11 Mesh Networks",

5.Amjad Ali, Muhammad Ejaz Ahmed, Md. Jalil Piran and Doug Young Suh, "Resource Optimization Scheme for Multimedia-Enabled Wireless Mesh Networks",Sensors,2014.

6.DEGAN ZHANG,YANAN ZHU,SI LIU,XIAODAN ZHANG AND JINJIE SONG, "Multi-Radio MultiChannel (MRMC) Resource Optimization Method for Wireless Mesh Network",JOURNAL OF INFORMATION SCIENCE AND ENGINEERING 32, 495-514,2016.

7.Yong Ding, Kanthakumar Pongaliur, and Li Xiao, "Channel Allocation and Routing in Hybrid Multichannel Multiradio Wireless Mesh Networks",IEEE TRANSACTIONS ON MOBILE COMPUTING, VOL. 12, NO. 2, FEBRUARY 2013.

8.Jihong Wang, Wenxiao Shi, Keqiang Cui, Feng Jin and Yuxin Li, "Partially overlapped channel assignment for multi-channel multi-radio wireless mesh networks",EURASIP Journal onWireless Communications and Networking (2015) 2015:25,2015.

9. Xin-She Yang and Xingshi He,” Firefly Algorithm: Recent Advances and Applications”,

10.Mansoor Alicherry Randeep Bhatia Li (Erran) Li, "Joint Channel Assignment and Routing for Throughput Optimization in Multiradio Wireless Mesh Networks", IEEE Journal on Selected Areas in Communications, Volume: 24 , Issue: 11 , Nov. 2006.

11.Jasmine David. D, Jegathesan V, Roopa Jayasingh.J, "Performance of Dynamic Priority Based Interference Aware Channel Allocation in Wireless Mesh Networks for Energy Conservation", International Journal of Innovative Technology and Exploring Engineering (IJITEE), Vol-9, No-1, 2019.

12.A. Hamed Mohsenian Rad and Vincent W.S. Wong, "Joint Optimal Channel Assignment and Congestion Control for Multi-channel Wireless Mesh Networks", IEEE International Conference on Communications, Turkey, 2006. 